\title{
Molecular Pathology
}

Editor

LAUREN L. RITTERHOUSE

\section{SURGICAL PATHOLOGY CLINICS}

www.surgpath.theclinics.com

Consulting Editor

JASON L. HORNICK

September 2021 • Volume 14 • Number 3 